\title{
Comment
}

Neuro epidemiology

Received: August 16, 2008

Neuroepidemiology 2009;32:80

DOI: $10.1159 / 000170911$

\section{Different Risk Factors in Vascular Dementia and Ischaemic Stroke}

Rahul Potluri a , Ammar Natalwala a , Hardeep Uppal ${ }^{\text {b, }}$ Reinhard Heun ${ }^{\mathrm{b}}$

aThe Medical School, University of Birmingham, and

${ }^{b}$ Department of Old Age Psychiatry, Queen Elizabeth

Psychiatric Hospital, Birmingham, UK

Vascular risk factors that predispose to vascular dementia and ischaemic stroke have recently been debated. We observed that vascular risk factors for vascular dementia and ischaemic stroke differ [1]; however, Thrift et al. [2] argued that differential misclassification, under-diagnosis of vascular dementia, the lack of a control group and the selection of variables may have confounded our results.

Misclassification cannot be ruled out in several thousand patients and many clinicians over a period of several years (20002006); however, there is no evidence that atrial fibrillation, hypertension or other risk factors were differentially diagnosed in vascular dementia and stroke, especially since ischaemic stroke is a major risk factor for vascular dementia (95.9\% in our sample) [1]. If misclassification differences were true, we should have severe concern in the care of patients with vascular dementia, as these patients are just as likely to suffer from future ischaemic strokes as ischaemic stroke patients themselves. In addition, secondary diagnoses, such as hyperlipidaemia and diabetes mellitus, were based on blood test results having identical thresholds for all patients; thus, preventing differential misclassification.

Vascular dementia was diagnosed using the ICD-10 classification, which has rigid specific criteria for patients to be labelled with vascular dementia. Therefore, there is some possibility that vascular dementia according to ICD-10 was under-diagnosed, as a considerable proportion of patients in the total sample were di- agnosed as unspecified dementia in some of our subsequent work [3]. Moreover, our conclusions resulted from the comparison of subjects with vascular dementia and age-matched control subjects, and thus should not be biased. In agreement with the proposal of Thrift et al. [2], our comparison group was indeed 95,734 age- and gender-matched control patients who were admitted to our large teaching hospital in Birmingham, UK, during the period 2000-2006 [1]

In concordance with Thrift et al. [2], we are fully aware that the selection of independent variables is paramount in logistic regression analysis. We can confirm that different models including or excluding stroke consistently revealed that vascular risk factors, including hypertension, diabetes mellitus and atrial fibrillation, were not significant risk factors for vascular dementia, even though they were significant risk factors for ischaemic stroke.

Consequently, we are very confident that our observation that risk factors in vascular dementia and ischemic stroke differ is valid [1]. Our results are of relevance as they challenge the unproven clinical assumption that risk factors as well as the pathophysiology of ischemic stroke and vascular dementia are identical. We are sorry that due to limitations of space we could not include all the relevant information in our recent letter [1].

\section{References}

1 Potluri R, Natalwala A, Uppal H, Heun R: Cardiovascular risk factors in vascular dementia and ischaemic stroke. Neuroepidemiology 2008; 31:67.

2 Thrift A, Srikanth VK: Risk factors for dementia. Neuroepidemiology 2008:31:68.

3 Natalwala A, Potluri R, Uppal H, Heun R: Reasons for hospital admissions in dementia patients in Birmingham, UK, during 2002-2007. Dement Geriatr Cogn Disord 2008, in press.

Prof. Reinhard Heun

Division of Neuroscience, Department of Psychiatry

Queen Elizabeth Psychiatric Hospital, Mindelsohn Way, Edgbaston Birmingham B15 2QZ (UK)

Tel. +44 121678 2360, Fax +44 1216782351

E-Mail r.heun@bham.ac.uk

\section{KARGER}

Fax +41613061234 E-Mail karger@karger.ch www.karger.com
(2) 2008 S. Karger AG, Base

0251-5350/09/0321-0080\$26.00/0

Accessible online at:

www.karger.com/ned 